\title{
Capacidad diagnóstica de la ecografía para detectar cáncer de endometrio en mujeres posmenopaúsicas sintomáticas y asintomáticas: experiencia de la Universidad Católica de Chile
}

\author{
Trinidad Raby B. ${ }^{1}$, Paula Sierra Valderrama. ${ }^{2}$, Harumi Tsunekawa O. $^{1, a}$, José Miguel \\ Craig V. ${ }^{1}$, Jorge Carvajal C. ${ }^{1}$, Mauricio Cuello F. $^{1}$ \\ ${ }^{1}$ División de Obstetricia y Ginecología, Facultad de Medicina, Pontificia Universidad Católica de Chile, ${ }^{2}$ Médico ciruja- \\ no, Pontificia Universidad Católica de Chile.
}

a Enfermera Matrona.

\section{RESUMEN}

Antecedentes: El 10\% de las mujeres postmenopáusicas con sangrado uterino anormal (SUA) tendrán cáncer de endometrio. Se recomienda efectuar una biopsia endometrial en toda mujer postmenopáusica con SUA y grosor endometrial ecográfico $>5 \mathrm{~mm}$. Estudios recientes sugieren que el uso de un valor menor (3 $\mathrm{mm}$ ) mejora la capacidad diagnóstica de la ecografía. En mujeres postmenopáusicas asintomáticas se ha sugerido efectuar biopsia endometrial si se detecta un endometrio $>11 \mathrm{~mm}$. Objetivo: Determinar la capacidad diagnóstica de la ecografía para detectar cáncer de endometrio, utilizando los valores de corte de 3 y $5 \mathrm{~mm}$ en mujeres posmenopáusicas sintomáticas y de $11 \mathrm{~mm}$ en mujeres postmenopáusicas asintomáticas. Método: Revisión retrospectiva de biopsias de endometrio e historia clínica de mujeres atendidas en la Red de Salud UC (2007-2012). Resultados: Se analizó 132 casos, 63,6\% presentaron SUA. Hubo 17 casos de cáncer de endometrio (12,9\%), con un grosor endometrial promedio de $18 \mathrm{~mm}$, el $88 \%$ presentó SUA. En mujeres sintomáticas la sensibilidad para el diagnóstico de cáncer de endometrio fue de $100 \%$ y de $93 \%$ para valores de corte 3 y $5 \mathrm{~mm}$ respectivamente. En mujeres asintomáticas, el punto de corte $11 \mathrm{~mm}$, tuvo una sensibilidad de $50 \%$ y una especificidad de $65 \%$ para el diagnóstico de cáncer de endometrio. Conclusión: Recomendamos efectuar biopsia endometrial a toda mujer postmenopáusica con SUA y endometrio $>3 \mathrm{~mm}$. En postmenopáusicas sin SUA, no recomendamos la evaluación ecográfica endometrial de rutina.

\section{PALABRAS CLAVES: Endometrio, ecografía, cáncer de endometrio, sangrado uterino anormal}

\section{SUMMARY}

Introduction: $10 \%$ of postmenopausal women with abnormal uterine bleeding (AUB) have endometrial cancer. Endometrial biopsy is recommended in all postmenopausal women with AUB and endometrial thickness $>5 \mathrm{~mm}$ on ultrasound. Recent studies suggest that the use of a lower value $(3 \mathrm{~mm})$ improves the ability of ultrasound to detect endometrial cancer. In asymptomatic postmenopausal women, endometrial biopsy is recommended if the endometrial thickness is $>11 \mathrm{~mm}$. Objective: To determine the diagnostic accuracy of ultrasound for detecting endometrial cancer, using a cutoff value of 3 and $5 \mathrm{~mm}$ in symptomatic and 11 $\mathrm{mm}$ in asymptomatic postmenopausal women. Methods: Retrospective review of endometrial biopsies and clinical history of women attending UC Health Network (2007-2012). Results: 132 cases were analyzed, $63.6 \%$ had AUB. There were 17 cases of endometrial cancer (12.9\%), with a mean endometrial thickness 
of $18 \mathrm{~mm} .88 \%$ of women with endometrial cancer had SUA. In symptomatic women the sensitivity for the diagnosis of endometrial cancer was $100 \%$ and $93 \%$ using a cutoff value of 3 and $5 \mathrm{~mm}$ respectively. In asymptomatic women, the cutoff value of $11 \mathrm{~mm}$, had a sensitivity of $50 \%$ and a specificity of $65 \%$ for endometrial cancer. Conclusion: We recommend endometrial biopsy in all postmenopausal women with AUB and endometrial thickness $>3 \mathrm{~mm}$. In postmenopausal women without AUB we do not recommend routine endometrial ultrasound evaluation.

\section{KEY WORDS: Endometrium, ultrasound, endometrial cancer, abnormal uterine bleeding}

\section{INTRODUCCIÓN}

El sangrado uterino anormal (SUA) es un problema frecuente en ginecología (1). EI SUA puede deberse a patología orgánica o funcional. Las patologías orgánicas más frecuente son: pólipo endometrial, mioma submucoso, hiperplasia endometrial y cáncer de endometrio (1). Se ha demostrado que en mujeres posmenopáusicas que presentan SUA, el 10\% tendrán un cáncer de endometrio (2), es por esto que existe gran interés en determinar cuál es la mejor manera de estudiar la etiología del SUA en estos casos.

El cáncer de endometrio ha aumentado su incidencia en las últimas décadas, siendo actualmente el primer cáncer de origen ginecológico en los países desarrollados (3). En Chile, su prevalencia es de 1,2 por 100.000 mujeres (4). El riesgo acumulado de padecer cáncer de endometrio a lo largo de la vida, es de $1,6 \%$ en países desarrollados y de $0,7 \%$ en los países subdesarrollados (5). La sobrevida del cáncer de endometrio es alta (86\%) cuando el diagnóstico se realiza precozmente, con la enfermedad limitada al útero (3).

La sospecha de cáncer de endometrio, se basa en la presencia de factores de riesgo, la clínica (SUA) y el aumento del grosor endometrial a la ecografía. Entre los factores de riesgo, destacan aquellos relacionados con altos niveles de estrógenos (ciclos anovulatorios y uso de estrógenos exógenos) (6), la obesidad y la nuliparidad (3).

La mayoría de las veces $(90 \%)$, el cáncer de endometrio se manifiesta tempranamente como SUA (7), sin embargo, sólo el $10 \%$ de las mujeres postmenopáusicas con SUA, tendrán cáncer de endometrio (2). Existen casos de cáncer de endometrio en mujeres asintomáticas (10\%), es decir, aquellas que no presentan SUA (7). Es importante destacar, que el cáncer de endometrio Tipo II (i.e. seroso papilar, carcinoma de células claras, carcinoma endometrial indiferenciado), pueden no manifestarse con SUA o endometrio patológico a la ecografía (8).
La medición del grosor endometrial en la ecografía, es una herramienta importante para la evaluación de las mujeres que consultan por SUA, especialmente para determinar si la paciente tiene alto o bajo riesgo de cáncer endometrial y así decidir si requiere o no una biopsia endometrial (9). La correcta interpretación del grosor endometrial en la ecografía, requiere considerar la edad de la paciente, el día del ciclo menstrual en que se realiza y el uso de THR, ya que las hormonas sexuales secretadas por el ovario o administradas de manera exógena, alteran considerablemente el grosor endometrial (10).

Existen varios estudios publicados sobre la relación que existe entre cáncer de endometrio y grosor endometrial ecográfico (11-14). Para mujeres posmenopáusicas con SUA, la sensibilidad y especificidad para el diagnóstico de cáncer de endometrio, con un grosor endometrial $\geq 4-5 \mathrm{~mm}$, es de $96 \%$ y $61 \%$ respectivamente (11-13). En efecto este valor ha sido clásicamente usado para decidir si hacer o no una biopsia endometrial en mujeres postmenopáusicas con SUA. Recientemente, otro grupo reportó que con un grosor endometrial de 3 $\mathrm{mm}$, la sensibilidad y especificidad para el diagnóstico de cáncer de endometrio en postmenopáusicas con SUA, era de $98 \%$ y $35 \%$ (14), recomendando el uso de $3 \mathrm{~mm}$ de grosor endometrial como valor de corte para decidir que mujeres requieren biopsia endometrial. Dado que el cáncer de endometrio tipo II, puede presentarse en un endometrio atrófico, ecográficamente muy delgado, algunos autores proponen que toda mujer postmenopáusica que presente SUA, debe ser sometida a un estudio histológico del endometrio (8).

Existen pocos estudios que evalúen el nivel de corte de grosor endometrial en mujeres postmenopáusicas asintomáticas. Se ha propuesto que un grosor endometrial $\geq 11 \mathrm{~mm}$, sería equivalente a tener un grosor endometrial de $5 \mathrm{~mm}$ en mujeres sintomáticas, con respecto al riesgo de tener un cáncer de endometrio (15). Sin embargo, no se recomienda el tamizaje con ecografía para el 
diagnóstico de cáncer de endometrio en mujeres asintomáticas, ya que la prevalencia de cáncer de endometrio en éste grupo es baja $(0,62 \%)(16)$.

Ante las opciones mostradas ( $3 \circ 5 \mathrm{~mm}$ en postmenopáusicas sintomáticas y $11 \mathrm{~mm}$ en postmenopáusicas asintomáticas), estimamos que se requiere determinar cuál es el grosor endometrial que permite seleccionar a las mujeres que se beneficiarán de una biopsia endometrial, específicamente disponer de un nivel de corte sobre el cual es necesaria la biopsia y bajo el cual es razonable un manejo expectante.

El objetivo de este trabajo, es determinar la capacidad diagnóstica de la ecografía respecto del cáncer de endometrio, utilizando los valores de corte de $3 \mathrm{~mm}$ y $5 \mathrm{~mm}$ en mujeres posmenopáusicas sintomáticas y de $11 \mathrm{~mm}$ en posmenopáusicas asintomáticas, atendidas en la Red de Salud UC.

\section{PACIENTES Y MÉTODOS}

Se realizó una revisión de la base de datos de pabellón y se analizaron los procedimientos quirúrgicos donde se obtuvo biopsia de endometrio en el Hospital Clínico de la Pontificia Universidad Católica de Chile, entre los años 2007-2012. Además, se revisó la base de datos del Servicio de Anatomía Patológica del mismo hospital, de donde se obtuvo los datos sobre las biopsias aspirativas de endometrio (ambulatorias) en ese mismo periodo de tiempo. Se hizo una revisión de la historia clínica de cada paciente mediante el sistema electrónico de fichas clínicas y la base de datos ecográficas. Se excluyeron mujeres cuya evaluación endometrial ecográfica fuera mayor a 3 meses antes de la obtención de la muestra y aquellas que no tuvieran registro completo de la historia clínica. El estudio fue aprobado por el Comité de Ética de la Facultad de Medicina de la Pontificia Universidad Católica de Chile.

\section{RESULTADOS}

Se detectó un total de 977 biopsias de endometrio. Estas biopsias, fueron obtenidas por diferentes métodos: biopsia aspirativa ( $n: 440)$, legrado uterino (n: 72), biopsia por histeroscopia (n: 340) y pieza quirúrgica de histerectomía (n: 125). De las 977 biopsias, 633 no fueron consideradas en esta investigación por no presentar estudio ecográfico registrado previo al procedimiento, por presentar más de 3 meses de desfase entre la ecografía y la biopsia y/o por no contar con la historia clínica completa de la paciente. Se analizó un total de 344 mujeres que tenían registro completo de los datos clínicos, grosor endometrial medido mediante ecografía y resultado de la biopsia endometrial.

La edad promedio de todas las mujeres fue de 47 años (rango: 18 a 84 años). De las 344 mujeres, $212(61,6 \%)$ eran premenopáusicas y $132(38,4 \%)$ posmenopáusicas, estas últimas constituyeron el grupo estudiado.

La edad promedio de las mujeres posmenopáusicas fue de 57 años (rango: 36 a 84 años). La mujer de 36 años tenía diagnóstico de menopausia prematura confirmada con estudio endocrinológico. De todas las posmenopáusicas, $84(63,6 \%)$ fueron sintomáticas (SUA). Hubo 19 (14,4\%) mujeres que usaban THR al momento de la evaluación y 12 $(9,0 \%)$ usuarias de tamoxifeno. En las posmenopáusicas con SUA, el 13,2\% eran usuarias de THR y el $7,2 \%$ usuarias de tamoxifeno. El grosor endometrial promedio de las mujeres posmenopáusicas fue de $21 \mathrm{~mm}$ (rango: 1-40 mm).

Dentro de los diagnósticos que incentivaron el estudio histológico, el principal fue la presencia de metrorragia en mujeres sintomáticas y sospecha de pólipo endometrial en la ecografía en las asintomáticas (Tabla I). Los diagnósticos de sospecha fueron aquellos que el cirujano registró como diagnóstico pre operatorio en el protocolo quirúrgico. El diagnóstico de sospecha "endometrio patológico" fue utilizada por los cirujanos para referirse a un endometrio engrosado (de acuerdo a la edad reproductiva) o de aspecto heterogéneo en la ecografía.

En la Tabla II, se señalan los diagnósticos histológicos de acuerdo a la presencia o ausencia de SUA en mujeres posmenopáusicas. El diagnóstico histológico más frecuente en todo el grupo fue el pólipo endometrial $(36,4 \%)$.

En la población estudiada, hubo un total de 17 cánceres de endometrio $(12,9 \%)$, con un grosor endometrial promedio de $18 \mathrm{~mm}$. Quince (88\%) se presentaron en posmenopáusicas con SUA y $2(12 \%)$ en aquellas sin SUA. Todos los casos de cáncer de endometrio se presentaron en mujeres con grosor endometrial mayor o igual a $3 \mathrm{~mm}$. Hubo un caso de cáncer de endometrio en una mujer cuyo grosor endometrial fue menor a $5 \mathrm{~mm}$. Se trató de una mujer posmenopáusica sintomática, a la cual se le realizó una histerectomía, con diagnóstico previo de cáncer de endometrio, el cual se realizó mediante la biopsia de un pólipo endometrial en expulsión; en la evaluación previa a la histerectomía, el grosor endometrial fue de $4 \mathrm{~mm}$. 
Tabla I

DIAGNÓSTICOS DE SOSPECHA PREVIO AL ESTUDIO ENDOMETRIAL EN POSMENOPÁUSICAS

\begin{tabular}{lrrr}
\hline Diagnóstico sospecha & $\begin{array}{r}\text { Total mujeres } \\
\mathrm{n}(\%)\end{array}$ & $\begin{array}{r}\text { Con SUA } \\
\mathrm{n}(\%)\end{array}$ & $\begin{array}{r}\text { Sin SUA } \\
\mathrm{n}(\%)\end{array}$ \\
\hline Pólipo & $50(37,9)$ & $23(27,4)$ & $27(56,3)$ \\
Metrorragia & $31(23,5)$ & $31(36,9)$ & $0(0)$ \\
Hiperplasia endometrial & $18(13,6)$ & $9(10,7)$ & $9(18,8)$ \\
Endometrio patológico & $16(12,1)$ & $9(10,7)$ & $7(14,6)$ \\
Cáncer de endometrio & $14(10,6)$ & $11(13,1)$ & $3(6,3)$ \\
Mioma & $2(1,5)$ & $1(1,2)$ & $1(2,0)$ \\
Colección intrauterina & $1(0,8)$ & $0(0)$ & $1(2,0)$ \\
TOTAL & $132(100)$ & $84(100)$ & $48(100)$ \\
\hline
\end{tabular}

Tabla II

DIAGNÓSTICO HISTOLÓGICOY GROSOR ENDOMETRIAL EN POSMENOPÁUSICAS CONY SIN SANGRADO UTERINO ANORMAL

\begin{tabular}{|c|c|c|c|c|}
\hline Diagnóstico histológico & $\begin{array}{r}\text { Total mujeres } \\
\mathrm{n}(\%)\end{array}$ & $\begin{array}{r}\text { Con SUA } \\
\mathrm{n}(\%)\end{array}$ & $\begin{array}{r}\text { Sin SUA } \\
n(\%)\end{array}$ & $\begin{array}{c}\text { Grosor endometrial } \\
\bar{x}(\mathrm{~mm})\end{array}$ \\
\hline Pólipo & $48(36,4)$ & $28(33,3)$ & $20(41,7)$ & 10 \\
\hline Hiperplasia sin atipia & $22(16,7)$ & $16(19,0)$ & $6(12,5)$ & 11 \\
\hline Cáncer de endometrio & $17(12,9)$ & $15(17,9)$ & $2(4,2)$ & 18 \\
\hline Endometrio atrófico & $15(11,4)$ & $9(10,7)$ & $6(12,5)$ & 11 \\
\hline Endometrio proliferativo & $11(8,3)$ & $7(8,3)$ & $4(8,3)$ & 10 \\
\hline Muestra insuficiente & $7(5,3)$ & $2(2,4)$ & $5(10,4)$ & 5 \\
\hline Mioma & $4(3,0)$ & $3(3,6)$ & $1(2,0)$ & 12 \\
\hline Otros & $4(3,0)$ & $2(2,4)$ & $2(4,2)$ & 15 \\
\hline Endometrio secretor & $2(1,5)$ & $0(0)$ & $2(4,2)$ & 16 \\
\hline \multirow[t]{2}{*}{ Hiperplasia con atipia } & $2(1,5)$ & $2(2,4)$ & $0(0)$ & 10 \\
\hline & $132(100)$ & $84(100)$ & $48(100)$ & 12 \\
\hline
\end{tabular}

Considerando como punto de corte los $5 \mathrm{~mm}$ en todas las posmenopáusicas (sintomáticas y asintomáticas), vemos que la incidencia de cáncer endometrial en las mujeres con grosor endometrial menor a $5 \mathrm{~mm}$ fue de $0,8 \%$, en comparación con un 12,1\% de cáncer de endometrio en mujeres con grosor endometrial $\geq 5 \mathrm{~mm}$.

En mujeres posmenopáusicas sintomáticas, todos los cánceres de endometrio fueron detectados al considerar como valor de corte los $3 \mathrm{~mm}$. Al considerar el valor de corte de $5 \mathrm{~mm}$, hubo un caso que no hubiese sido diagnosticado (Tabla III).

Para el diagnóstico de patología maligna y pre maligna, es decir, cáncer de endometrio e hiperplasia endometrial con atipia, se analizó la sensibilidad y especificidad para los valores de corte de $3 \mathrm{~mm}$ y $5 \mathrm{~mm}$ (Tabla IV).

Entre las posmenopáusicas sin SUA (asintomáticas), hubo 2 casos de cáncer de endometrio, con un grosor endometrial promedio de $10 \mathrm{~mm}$ (rango: 5 a $14 \mathrm{~mm}$ ). En las mujeres postmenopáusicas sin SUA el uso de un valor de corte de $11 \mathrm{~mm}$ para el diagnóstico de cáncer de endometrio, mostró baja sensibilidad (Tabla V).

Entre las mujeres postmenopáusicas sometidas a estudio endometrial por colección intracavitaria ( $\mathrm{n}$ : 11), el $36 \%$ presentó SUA. Hubo 3 casos de cáncer de endometrio en mujeres con colección intracavitaria, todos se presentaron en postmenopáusicas con SUA y el grosor endometrial promedio fue de $16 \mathrm{~mm}$ 
(rango: 5 a $40 \mathrm{~mm}$ ). En postmenopáusicas usuarias de tamoxifeno, hubo sólo un caso de cáncer de endometrio. La razón para realizar el estudio, fue la presencia de SUA asociado a un endometrio de 14 $\mathrm{mm}$. En usuarias de THR hubo sólo 2 casos de cáncer de endometrio, con un grosor endometrial de 5 y $15 \mathrm{~mm}$; ambas eran usuarias de THR combinada continua, una mujer presentó SUA, mientras la otra fue asintomática.

\section{DISCUSIÓN}

En nuestro estudio, la sensibilidad para el diagnóstico de cáncer de endometrio en mujeres posmenopáusicas con SUA fue de $100 \%$ al considerar como punto de corte los $3 \mathrm{~mm}$ y de $93 \%$ al considerar como punto de corte los $5 \mathrm{~mm}$. Resultados similares se observan al agrupar la patología maligna y pre maligna en posmenopáusicas con SUA. En el grupo de mujeres sin SUA, observamos una sensibilidad de $50 \%$ y una especificidad de $65 \%$ para el diagnóstico de cáncer de endometrio usando como valor de corte los $11 \mathrm{~mm}$.

Actualmente, no existe consenso sobre cual nivel de corte de grosor endometrial se debe usar en posmenopáusicas con SUA para determinar la necesidad de estudio histológico y existen pocos trabajos nacionales que relacionan el grosor endometrial y patología asociada (17). En mujeres posmenopáusicas con SUA, se ha reportado una sensibilidad para el diagnóstico de cáncer de endometrio de $96 \%$ tomando como nivel de corte los $5 \mathrm{~mm}$ (11) y de $97,9 \%$ para un corte de $3 \mathrm{~mm}$ (14). La mayoría de los autores, está de acuerdo con utilizar 4-5 mm como punto de corte (11), sin embargo, existen otros estudios que demuestran

Tabla III

CAPACIDAD DIAGNÓSTICA DEL GROSOR ENDOMETRIAL EN POSMENOPÁUSICAS CON SANGRADO UTERINO ANORMAL: CÁNCER DE ENDOMETRIO

\begin{tabular}{lcccccc}
\hline Valor de corte & S (\%) & E (\%) & VPP (\%) & VPN (\%) & LR (+) & LR (-) \\
\hline$\geq 3 \mathrm{~mm}$ & 100,0 & 6,0 & 19,0 & 100,0 & 1,06 & 0,00 \\
$\geq 5 \mathrm{~mm}$ & 93,0 & 13,0 & 19,0 & 90,0 & 1,07 & 0,51 \\
\hline
\end{tabular}

S: sensibilidad. E: Especificidad. VPP: valor predictivo positivo. VPN: valor predictivo negativo. LR: likelihodd-ratio.

\section{Tabla IV}

CAPACIDAD DIAGNÓSTICA DEL GROSOR ENDOMETRIAL EN POSMENOPÁUSICAS CON SANGRADO UTERINO ANORMAL: PATOLOGÍA MALIGNA Y PRE MALIGNA

\begin{tabular}{lcccccc}
\hline Valor de corte & $S(\%)$ & $E(\%)$ & VPP (\%) & VPN (\%) & LR (+) & LR (-) \\
$\geq 3 \mathrm{~mm}$ & 100,0 & 6,0 & 21,0 & 100,0 & 1,06 & 0,00 \\
$\geq 5 \mathrm{~mm}$ & 94,0 & 13,0 & 22,0 & 90,0 & 1,09 & 0,44 \\
\hline
\end{tabular}

S: sensibilidad. E: Especificidad. VPP: valor predictivo positivo. VPN: valor predictivo negativo. LR: likelihodd-ratio.

Tabla V

CAPACIDAD DIAGNÓSTICA DEL GROSOR ENDOMETRIAL EN POSMENOPÁUSICAS SIN SANGRADO UTERINO ANORMAL: CÁNCER DE ENDOMETRIO

\begin{tabular}{lcccccc}
\hline Valor de corte & $S(\%)$ & $E(\%)$ & VPP (\%) & VPN (\%) & LR (+) & LR (-) \\
\hline$\geq 11 \mathrm{~mm}$ & 50 & 65 & 6 & 97 & 1,44 & 0,77
\end{tabular}

S: sensibilidad. E: Especificidad. VPP: valor predictivo positivo. VPN: valor predictivo negativo. LR: likelihodd-ratio. 
que puede haber cáncer de endometrio incluso en mujeres con endometrio menor a $3 \mathrm{~mm}(9,18)$. En general, dicho fenómeno se observa en cáncer de endometrio tipo II, que si bien es menos frecuente (10-15\%), da cuenta del $50 \%$ de la mortalidad por cáncer de endometrio (8).

En nuestro estudio, sólo un caso de cáncer de endometrio se presentó con endometrio menor a $5 \mathrm{~mm}$ (en este caso el grosor endometrial fue de $4 \mathrm{~mm}$ ). El caso correspondió a una paciente con cáncer de endometrio poco diferenciado de células claras, cuyo diagnóstico inicial se realizó mediante la biopsia de un pólipo endometrial en expulsión y cuya ecografía antes de la histerectomía mostró un endometrio delgado. Este caso ilustra el comportamiento de los cánceres de endometrio tipo II. Como podemos ver en este caso, un endometrio delgado a la ecografía no descarta la presencia de cáncer de endometrio y creemos que toda mujer postmenopáusica requiere de un estudio endometrial histológico para determinar la causa del sangrado, aún con endometrio delgado a la ecografía.

El año 2004 se publicó un trabajo que propuso que en posmenopáusicas asintomáticas ( $\sin$ SUA), un grosor endometrial mayor o igual a $11 \mathrm{~mm}$ otorgaría el mismo riesgo de padecer cáncer de endometrio que en mujeres con SUA y endometrio mayor o igual a $5 \mathrm{~mm}$ (15). En nuestro estudio, al evaluar dicho corte de grosor endometrial $(11 \mathrm{~mm})$ en posmenopáusicas sin SUA, la ecografía no fue capaz de discriminar entre aquellas con y sin cáncer de endometrio. Nuestros resultados avalan la recomendación internacional de no realizar tamizaje ecografico en mujeres posmenopáusicas asintomáticas para la pesquisa de cáncer de endometrio (16), por su baja incidencia.

En nuestro estudio, 11 mujeres tenían el diagnóstico de colección intracaviaria en la ecografía. Tres de ellas $(27,2 \%)$ presentaron cáncer de endometrio. Todas presentaron SUA al momento de la evaluación y el grosor endometrial promedio fue de $16 \mathrm{~mm}$. La presencia de colección intracavitaria por sí sola, no involucra mayor riesgo de cáncer de endometrio, salvo que se asocie a un endometrio engrosado (19). Nuestros datos apoyan esta conclusión, ya que las 3 mujeres tenían indicación de biopsia endometrial dado su sintomatología y la asociación con endometrio engrosado, independiente si tuvieron o no colección intracavitaria al momento de la evaluación.

En cuanto al uso de tamoxifeno, el tamaño muestral no permite obtener conclusiones relevantes. Sin embargo, cabe destacar que en cuanto al uso de tamoxifeno, el Colegio Americano de
Ginecólogos y Obstetras (ACOG) no recomienda el uso rutinario de biopsia endometrial frente al hallazgo de endometrio engrosado a la ecografía en una mujer asintomática (20). Es así, como de las 12 usuarias de tamoxifeno en nuestro estudio, sólo una de ellas presentó cáncer de endometrio. Esta paciente presentó endometrio engrosado (14 $\mathrm{mm}$ ) y SUA, por lo tanto, tenía indicación de biopsia endometrial independiente si era o no usuaria de tamoxifeno. Este dato es importante, ya que en usuarias de tamoxifeno, es común encontrar endometrio engrosado y heterogéneo a la ecografía, pero dicho hallazgo no se asocia a mayor riesgo de cáncer de endometrio en mujeres asintomáticas (20). No recomendamos el uso de biopsia endometrial de rutina en usuarias de tamoxifeno, salvo que sean pacientes sintomáticas.

Entre nuestras mujeres con cáncer de endometrio, sólo dos eran usuarias de THR. Un grupo de nuestro centro, publicó la incidencia de patología endometrial en usuarias de THR, encontrando incidencia baja (1\%) de cáncer de endometrio (21). Al igual que en nuestro estudio, las mujeres eran usuarias de THR combinada; es esperable que la presencia del progestágeno en la TRH impida el aumento en el riesgo de cáncer de endometrio en relación a las no usuarias de THR.

Debemos mencionar, que nuestro estudio, producto de su diseño, presenta ciertas limitaciones. Tratándose de un estudio retrospectivo, es habitual la pérdida de información, especialmente en aquellas mujeres cuyo estudio se realizó de manera ambulatoria. En este caso, el mal registro hizo que tuviéramos que eliminar una cantidad considerable de las biopsias endometriales. Al tratarse de un reporte de casos, el evento de interés (cáncer de endometrio) es maximizado. Suponemos, que en el estudio se encuentra una mayor incidencia de cáncer de endometrio entre las posmenopáusicas con SUA, ya que consideramos a aquellas mujeres que tuvieron biopsia endometrial y no al universo completo de posmenopáusicas con SUA. Muchas posmenopáusicas con SUA y endometrio menor a $5 \mathrm{~mm}$, no fueron sometidas a biopsia endometrial y por lo tanto, no fueron incluidas en nuestro análisis. Además, se incluyeron a aquellas mujeres que habían sido sometidas a estudio endometrial por diferentes causas, sin un protocolo definido, es decir, la decisión de realizar el estudio estuvo basado en el juicio clínico de cada médico. Por lo tanto, los cálculos de sensibilidad y especificidad que presentamos no son aplicables a cualquier población de posmenopáusicas con SUA. 


\section{CONCLUSIÓN}

Ambos valores de corte ( 3 y $5 \mathrm{~mm}$ ) en posmenopáusicas con SUA presentan una sensibilidad aceptable, sin embargo, utilizando el valor de corte de $5 \mathrm{~mm}$, un caso de cáncer de endometrio no hubiera sido diagnosticado. Recomendamos efectuar una biopsia endometrial en toda mujer posmenopáusica que presente SUA y endometrio mayor 0 igual a $3 \mathrm{~mm}$. En mujeres sin SUA, la ecografía no permite discriminar entre aquellas que tienen más riesgo de cáncer de endometrio, por lo tanto, no recomendamos el tamizaje con ecografía transvaginal de rutina en mujeres postmenopáusicas sin SUA. Recomendamos la biopsia endometrial en mujeres con colección intracavitaria, solo cuando el endometrio sea mayor a $3 \mathrm{~mm}$ y presenten SUA.

\section{REFERENCIAS}

1. Ely JW, Kennedy CM, Clark EC, Bowdler NC. Abnormal uterine bleeding: a management algorithm. J Am Board Fam Med 2006;19(6):590-602.

2. Klarlsson B, Grangerg S, Wikland M, Ylostalo P, Torvid K, Marsal K, Valentin L. Transvaginal ultrasonography of the endometrium in women with postmenopausal bleeding--a nordic multicenter study. Am J Obstet Gynecol 1995;172(5):1488-94.

3. Arora V, Quinn M a. Endometrial cancer. Best Pract Res Clinical Obstet Gynaecol 2012;26(3):311-24.

4. Medina E, Kaempffer AM. Mortalidad por cáncer en Chile: consideraciones epidemiológicas. Rev Med Chile 2001;129(10):1195-202.

5. Jemal A, Bray F, Ferlay J. Global Cancer Statistics 2011;61(2):69-90.

6. Denschlag D, Ulrich U, Emons G. The diagnosis and treatment of endometrial cancer: progress and controversies. Dtsch Ärztebl Int 2010;108(34-35):571-7.

7. Managment of endometrial cancer. ACOG practice bulleting number 65. American College of Obstetricians and Gynecologist. 2005;106:413-25.

8. Wang J, Wieslander C, Hansen G, Cass I, Vasilev S, Holschneider $\mathrm{CH}$. Thin endometrial echo complex on ultrasound does not reliably exclude type 2 endometrial cancers. Gynecol Oncol 2006;101(1):120-5.

9. Haenggi W, Raio L. Ultrasound: a useful tool?. International Congress Series 2002;1229:163-70.
10. Gormaz G. Cambios ultrasonográficos del endometrio durante el ciclo menstrual. Rev Chil Obstet Ginecol1992;57:257-62.

11. Smith-Bindman $R$, Kerlikowske $K$, Feldstein VA, Subak L, Scheidler J, Segal M, et al. Endovaginal ultrasound to exclude endometrial cancer and other endometrial abnormalities. JAMA 1998;280(17):151017.

12. Tabor A, Watt HC, Wald NJ. Endometrial thickness as a test for endometrial cancer in women with postmenopausal vaginal bleeding. Obstet Gynecol 2002;99(4):663-70.

13. Gupta JK, Chien PFW, Voit D, Clark TJ, Khan KS. UItrasonographic endometrial thickness for diagnosing endometrial pathology in women with postmenopausal bleeding: a meta-analysis. Acta Obstet Gynecol Scand 2002;81(9):799-816.

14. Timmermans A, Opmeer B, Khan K, et al. Endometrial thickness measurement for detecting endometrial cancer in women with postmenopausal bleeding. Obstet Gynecol 2010;116(1):160-7.

15. Smith-Bindman R, Weiss E, Feldstein V. How thick is too thick? When endometrial thickness should prompt biopsy in postmenopausal women without vaginal bleeding. Ultrasound Obstet Gynecol 2004;24(5):558-65.

16. Breijer MC, Peeters JAH, Opmeer BC, Clark TJ, Verheijen RHM, Mol BWJ, et al. Capacity of endometrial thickness measurement to diagnose endometrial carcinoma in asymptomatic postmenopausal women: a systematic review and meta-analysis. Ultrasound Obstet Gynecol 2012;40(6):621-9.

17. Socias M, Vargas R, Costoya A, Monje B, Haye MT. Correlación de la histeroscopia y biopsia dirigida en el estudio del engrosamiento endometrial patológico por ultrasonido. Rev Chil Obstet Ginecol 2007;72(2):99104.

18. Naftalin J, Nunes N, Hoo W, Arora R, Jurkovic D. Endometrial cancer and ultrasound: why measuring endometrial thickness is sometimes not enough. UItrasound Obstet Gynecol 2012;39(1):106-9.

19. Inceboz U, Uyar Y, Baytur Y, Kandiloglu AR. Endometrial fluid in postmenopausal women. Int J Gynaecol Obstet 2009;107(2):154-5.

20. Tamoxifen and uterine cancer. ACOG Commitee Opinion No. 336. American College of Obstetricians and Gynecologist 2006;107:1475-8.

21. Bianchi M, Arteaga E, Villaseca P. Baja incidencia de patología endometrial en mujeres postmenopaúsicas con sangrado anormal que reciben terapia hormonal de reemplazo. Rev Chil Obstet Ginecol 2002;67(2):136-8. 